\title{
Influences on the uptake of health and wellbeing apps and curated app portals: a think aloud and interview study.
}

Dorothy Szinay, Olga Perski, Andy Jones, Tim Chadborn, Jamie Brown, Felix Naughton

Submitted to: JMIR mHealth and uHealth

on: January 14, 2021

Disclaimer: () The authors. All rights reserved. This is a privileged document currently under peer-review/community review. Authors have provided JMIR Publications with an exclusive license to publish this preprint on it's website for review purposes only. While the final peer-reviewed paper may be licensed under a CC BY license on publication, at this stage authors and publisher expressively prohibit redistribution of this draft paper other than for review purposes. 


\section{Table of Contents}

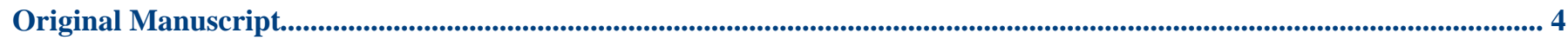

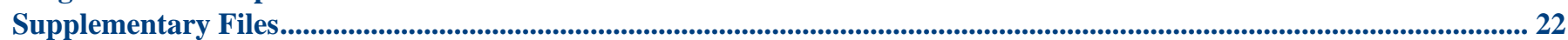

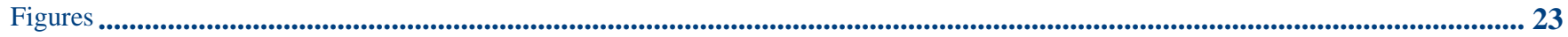

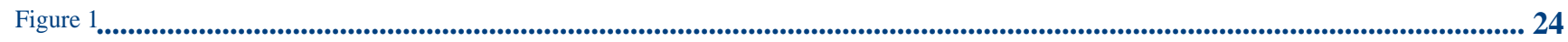

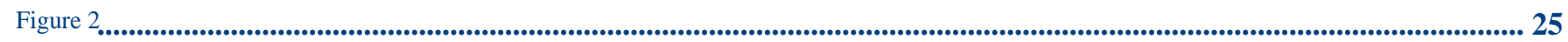

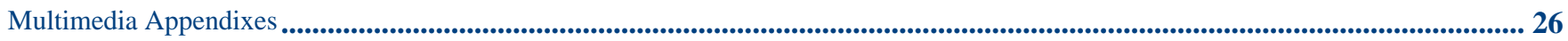

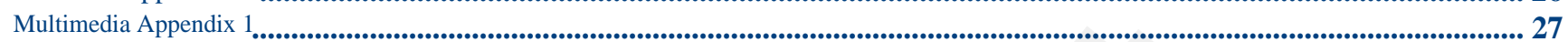

Multimedia Appendix 2

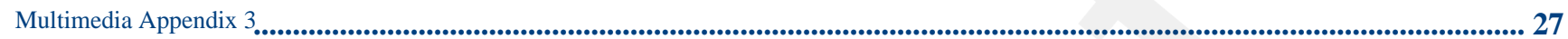

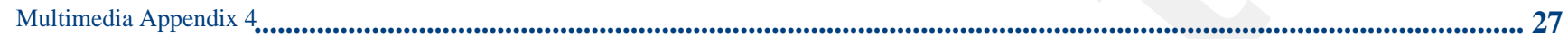




\section{Influences on the uptake of health and wellbeing apps and curated app portals: a think aloud and interview study.}

Dorothy Szinay ${ }^{1} \mathrm{MSc}$; Olga Perski ${ }^{2} \mathrm{PhD}$; Andy Jones ${ }^{1} \mathrm{PhD}$; Tim Chadborn ${ }^{3} \mathrm{PhD}$; Jamie Brown ${ }^{2} \mathrm{PhD}$; Felix Naughton ${ }^{1} \mathrm{PhD}$

\footnotetext{
${ }^{1}$ University of East Anglia Norwich GB

${ }^{2}$ University College London London GB

${ }^{3}$ Public Health England London GB
}

\section{Corresponding Author:}

Dorothy Szinay MSc

University of East Anglia

Norwich Research Park

Norwich

GB

\section{Abstract}

Background: Health and wellbeing smartphone apps can be identified through different routes, including via curated health app portals, but little is known about people's experiences of this.

Objective: This study explored how people select health apps online and their views on curated portals.

Methods: Eighteen UK-based adults were recruited and asked to verbalise their thoughts whilst searching for a health or wellbeing app online, including on two curated health app portals. This was followed by semi-structured interviews. Data were analysed using Framework Analysis, informed by the COM-B model and the Theoretical Domains Framework.

Results: Searching for health and wellbeing apps online was described as a 'minefield'. App uptake appeared to be influenced by i) capabilities (e.g. app literacy skills, health and app awareness), ii) opportunities (e.g. app aesthetics, cost and social influences) and iii) motivation (e.g. the perceived utility and accuracy of the app, and transparency about data protection). Social influences and the percieved utility of an app, in particular, were important. People were not previously aware of curated portals but found the concept appealing and likely to engender trust and address data protection concerns. While apps listed on these were perceived as more trustworthy, their presentation was considered disappointing.

Conclusions: The uptake of health and wellbeing apps appear primarily influenced by social influences and the perceived utility of the app. With curated health app portals perceived as credible, app uptake via such portals may mitigate concerns related to data protection and accuracy, but their implementation must better meet user needs. Clinical Trial: NA

(JMIR Preprints 14/01/2021:27173)

DOI: https://doi.org/10.2196/preprints.27173

\section{Preprint Settings}

1) Would you like to publish your submitted manuscript as preprint?

\section{$\checkmark$ Please make my preprint PDF available to anyone at any time (recommended).}

Please make my preprint PDF available only to logged-in users; I understand that my title and abstract will remain visible to all users. Only make the preprint title and abstract visible.

No, I do not wish to publish my submitted manuscript as a preprint.

2) If accepted for publication in a JMIR journal, would you like the PDF to be visible to the public?

$\checkmark$ Yes, please make my accepted manuscript PDF available to anyone at any time (Recommended).

Yes, but please make my accepted manuscript PDF available only to logged-in users; I understand that the title and abstract will remain Yes, but only make the title and abstract visible (see Important note, above). I understand that if I later pay to participate in <a href="http 


\section{Original Manuscript}




\section{Title}

Influences on the uptake of health and wellbeing apps and curated app portals: a think aloud and interview study.

\section{Authors}

Dorothy Szinay ${ }^{1} \mathrm{MSc}$, Olga Perski ${ }^{2} \mathrm{PhD}$, Andy Jones ${ }^{3} \mathrm{PhD}$, Tim Chadborn ${ }^{4} \mathrm{PhD}$, Jamie Brown ${ }^{2,5} \mathrm{PhD}$ Felix Naughton ${ }^{1}$ $\mathrm{PhD}$

${ }^{1}$ School of Health Sciences, University of East Anglia, Norwich Research Park, Norwich NR4 7TJ

${ }^{2}$ Department of Behavioural Science and Health, University College London, 1-19 Torrington Place, London WC1E 6BT

${ }^{3}$ Norwich Medical School, University of East Anglia, Norwich Research Park, Norwich NR4 7TJ

${ }^{4}$ Behavioural Insights, Public Health England, Wellington House, 133-155 Waterloo Road, London SE1 8UG

${ }^{5}$ SPECTRUM Consortium, London, UK

\section{Corresponding author:}

Dorothy Szinay, MSc

School of Health Sciences, University of East Anglia Norwich research park, Norwich NR4 7TJ

Room 1.27, Edith Cavell Building

d.szinay@uea.ac.uk

Twitter: @DorothySzinay 


\section{Abstract}

Background: Health and wellbeing smartphone apps could provide a cost-effective solution to addressing unhealthy behaviours. The selection of these apps tends to occur in commercial app stores, where thousands of health apps are available. Their uptake is often influenced by popularity indicators. However, these indicators are not necessarily associated with app effectiveness and evidence-based content. Alternative routes to app selection are increasingly available, such as via curated app portals, but little is known about people's experiences of them.

Objectives: To explore how people select health apps online and their views on curated app portals.

Methods: Eighteen UK-based adults were recruited through social media and asked during an in-person meeting to verbalise their thoughts whilst searching for a health or wellbeing app online on a platform of their choice, then repeat the search on two curated health app portals: the 'NHS Apps Library', and the Public Health England 'One You' App portal . This was followed by a semi-structured interview. Data were analysed using Framework Analysis, informed by the Capability, Opportunity, Motivation - Behaviour (COM-B) model and the Theoretical Domains Framework.

Results: Searching for health and wellbeing apps online was described as a 'minefield'. App uptake appeared to be influenced by participants' capabilities, such as app literacy skills, health and app awareness, and opportunities, including the availability of apps, app aesthetics, the price of an app and social influences. Motivation factors that seemed to affect uptake were perceived competence, time efficiency, the perceived utility and accuracy of the app, transparency about data protection, commitment and social identity, and a wide range of emotions. Social influences and the perceived utility of an app were highlighted as particularly important. Participants were not previously aware of curated portals but found the concept appealing. Curated heath app portals appeared to engender trust and alleviate data protection concerns. While apps listed on these were perceived as more trustworthy, their presentation was considered disappointing. This disappointment seemed to stem from the functionality of the portals, the lack of user guidance and lack of tailored content to an individual's needs.

Conclusions: The uptake of health and wellbeing apps appear to be primarily affected by social influences and the perceived utility of an app. App uptake via curated health app portals perceived as credible may mitigate concerns related to data protection and accuracy, providing their implementation meets user needs and expectations.

\section{Keywords}

Behaviour change, health apps, mhealth, smartphone app, framework analysis, COM-B, TDF, think aloud 


\section{Introduction \\ Background}

Noncommunicable diseases (e.g. diabetes, heart disease, cancer, poor mental health), are considered key threats to global health [1], and are driven by factors such as physical inactivity, poor diet, tobacco smoking, and excessive alcohol consumption. A key global public health policy priority is to enact policies to ensure the best possible health is available for all [2]. In the United Kingdom (UK), aims of the National Health Service (NHS) long term plan [3] and priorities of UK Government executives agencies such as Public Health England (PHE) are to provide a smoke-free society, to encourage healthier diets and to improve mental health [4]. Encouraging the use of digital health interventions, such as smartphone apps, may be one (cost-)effective way of contributing.

Health and wellbeing smartphone apps can be cost-effective solutions for changing health behaviours [5, 6]. Such tools can act as ideal platforms to deliver behaviour change interventions [7] because of their availability, portability and easy access [8]. Research has demonstrated early evidence of effectiveness of smartphone apps for smoking cessation [9], healthy dietary and physical activity promotion [5, 10-12], weight loss [5, 13, 14], alcohol reduction among non-dependent drinkers [15] and mental health promotion [16]. In addition, health apps can reach those resistant to help-seeking in person (e.g. due to stigma) by improving access to behaviour change interventions [17]. However, low uptake and poor engagement over time compromise the potential of health and wellbeing apps.

'Uptake' refers to the decision to select and install a health app [18]. The search for and selection of health apps tends to take place in commercial app stores, such as Google Play for android operating systems and the Apple App Store for iOS $[10,19]$. Thousands of health and wellbeing smartphone apps are available in the major app stores, a number that continues to grow [7] Research shows that the uptake of apps from commercial app stores tends to be influenced by indicators of popularity, such as the app's rank order, ratings and/or reviews, and its total number of downloads [19]. However, such popularity indicators are not necessarily positively associated with app effectiveness [20], and indeed may even be negatively related [21]. An associated problem with app uptake is that the vast majority of apps listed in commercial stores lack evidence about their efficacy [22] or effectiveness [23]. The need for quality marks in commercial app stores has been raised [24], as well as the need for regulation of health apps and evidence for their effectiveness [16]. Better transparency in an app's description to help people make an informed choice, including how the user's data are handled, how the app was developed, benefits explained in lay terms, as well as descriptions of the app content has been recommended [25-27].

A barrier to the uptake of evidence-informed apps is that not all apps are available to the public, or prominently displayed, via commercial app stores [22, 24]. Therefore, fewer people may benefit from available high-quality tools. Evidenceinformed apps tend to be promoted within community or health care settings (often targeting a specific geographic region/ country), or on curated health app 'portals'. These portals are websites presenting a list of selected health apps [28]. Health app portals can be government-funded, such as the UK National Health Service's 'Apps Library' or Public Health England's 'One You Apps' portal, or curated by private organisations, such as 'App Script' by IQVIA in the United States, the UK and the United Arab Emirates, the 'MyHealthApps' by PatientView's in Europe and the UK, or 'ORCHA' in the UK. These organisations can lend credibility to and have the potential to promote the uptake of selected health apps [29] by providing a list of safe, evidence-informed, tested and, where possible, clinically effective health apps for the general public to choose from.

Research has focused on the identification of factors that influence uptake of health apps in commercial app stores. There is an urgent need to explore whether the general public would be willing to use curated health app portals, which could improve the uptake of evidence-informed health and wellbeing apps [18]. Despite this need, little is known about views on curated health app portals. This study aimed to explore potential users' views on factors influencing the uptake of health apps in general, and on curated health app portals in particular, using think aloud and interview methodology.

\section{Theoretical framework}

The COM-B (Capability, Opportunity, Motivation - Behaviour) model [30] offers a comprehensive framework for understanding behaviour. In the context of the current research, the behaviour of interest is the uptake of health and wellbeing apps. The model proposes that behaviour arises due to the interaction of three components: capability (physical and psychological), opportunity (physical and social) and motivation (automatic and reflective). The Theoretical Domains Framework (TDF) [31], which contains 14 domains that can be mapped onto the components of the COM-B model, was also used. Together, the COM-B model and the TDF allow for a detailed analysis of data and identification of key factors influencing uptake in general and on curated health app portals in particular (Figure 1).

\section{Aims}

This qualitative study applied a theoretical framework informed by the COM-B model and the TDF to explore 1) factors influencing potential users' uptake of health and wellbeing smartphone apps through online searching and 2) their views on available curated health app portals. 


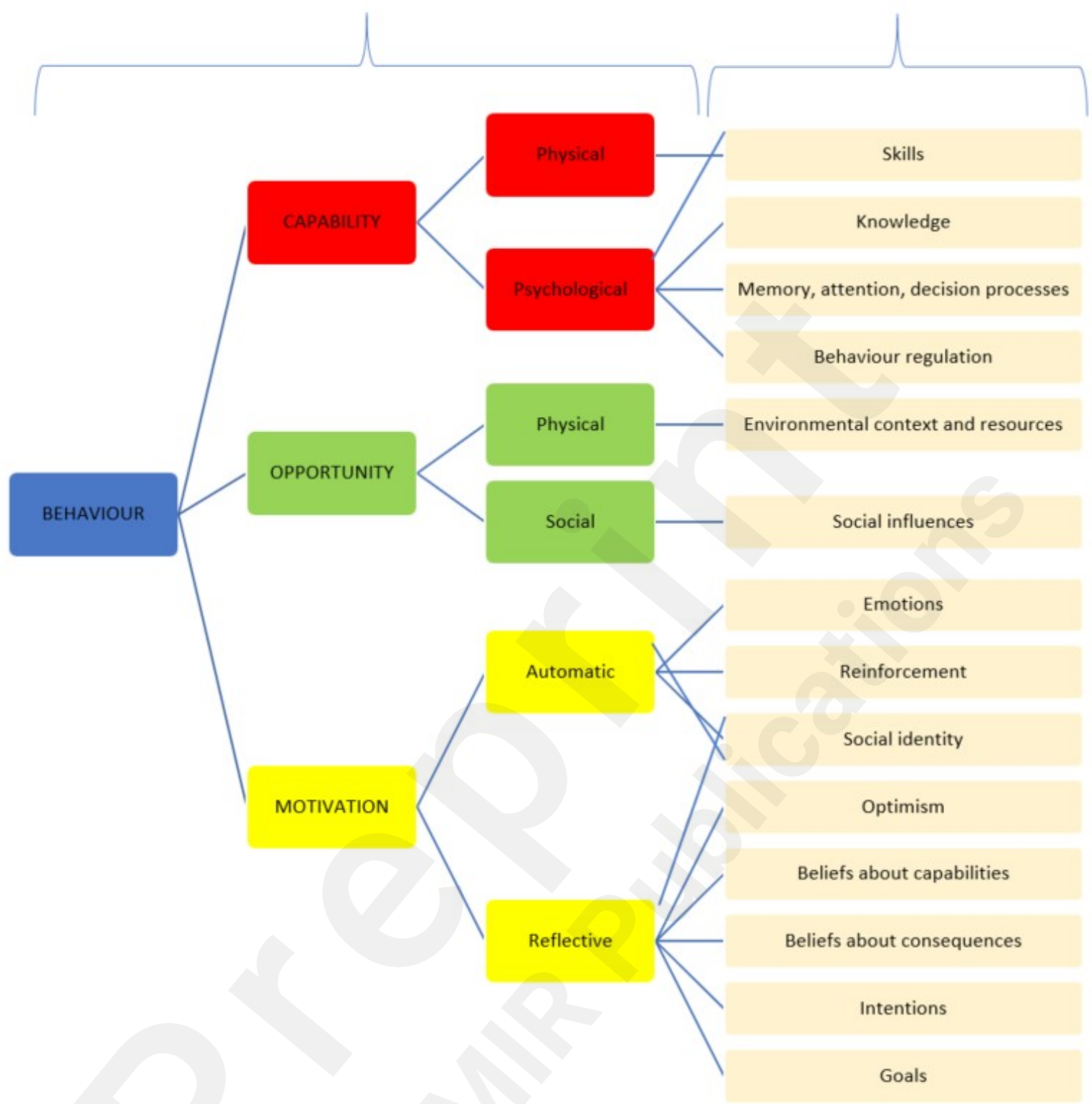

Figure 1. A visual representation of mapping the Theoretical Domains framework onto the COM-B model[18]

\section{Methods}

\section{Study design}

This research elicited views and preferences of a sample of members of the public. The Consolidated Criteria for Reporting Qualitative Research (COREQ) checklist guided the design of the study [32]. See the checklist in Multimedia Appendix 1. Think aloud methodology [33] was applied to collect real-time data about online health app selection, and involves asking participants to verbalise their thoughts and impressions throughout the selection process. The researcher only intervened when a prompt was considered necessary (e.g. during silent moments, asking questions such as 'What are you thinking now?). Following the think aloud tasks, follow-up questions were asked to better understand statements/utterances made during the tasks. Finally, semi-structured interview techniques were used. The think aloud tasks and the topic guide were informed by stakeholder consultation which included views and opinion of lay persons (patient and public involvement representatives) and expert opinion of policy makers of this research. The study protocol was pre-registered on the Open Science Framework [34]. The Faculty of Medicine and Health Sciences Ethics Committee at the University of East Anglia approved this study (Reference number: 201819 - 089). The collected data is stored following the European Union General Data Protection Regulation (GDPR) and the University of East Anglia Research Data Management Policy. The data was anonymised, and all personal identifiers were removed. All participants read the participant information sheet and provided consent prior taking part in this study. 


\section{Participants and recruitment}

Participants were recruited through paid advertisements on Facebook. Adults in the general population were eligible if they 1) were aged 18 or over; 2) were able to provide consent; 3) owned a smartphone; 4) would consider using a smartphone app to change their behaviour in the future; 5) were able to attend an interview in Norwich, England, where the work took place. As a standard practice in qualitative research, the aim of the study was to gain better understanding of the phenomenon of interest and to increase the coverage of perspectives rather than to necessarily recruit a populationrepresentative sample [35]. Hence, purposive sampling was used to promote the diversity of the sample (i.e. age, gender, ethnicity, educational level, employment) [36]. This included targeted adverts on Facebook and selection of participants to ensure the diversity of the sample. 114 individuals responded to the Facebook adverts and read a brief participant information sheet and completed the screening questionnaire. Out of 38 participants invited to an interview, 14 did not respond and 24 agreed to participate. Six of these 24 cancelled for various reasons.

\section{Procedure}

Prior to completing the online screening survey, participants were asked to read a brief participant information sheet describing the study. Once read and agreed to participate, participants were asked to complete an online questionnaire to assess their eligibility and to collect descriptive data (see Multimedia Appendix 2.). Data were collected on 1) age, 2) gender, 3) ethnicity, measured using the Office for National Statistics' index, 4) level of education, 5) employment status, 6) whether they have ever used health or wellbeing app, 7) whether they currently use a health or wellbeing app, 8) last time they had downloaded an app, and 9) frequency of app use. Participants who met the inclusion criteria were sent an email with a comprehensive participant information sheet and were invited for an interview. On the day of the interview, interviewees received a printed copy of the participant information sheet, and written consent was obtained.

Face-to-face interviews were conducted between July and August 2019 and took place at the University of East Anglia $(n=17)$ or the participants' home in Norwich $(n=1)$. The interviews were conducted by a single female researcher (DS) and no one else was present during the sessions. The session started with a think aloud exercise, with participants being instructed on how to verbalise their thoughts. First, they were asked to perform a search for an app they would potentially use to change a health behaviour of their choice. They had a choice of using either a study laptop or their smartphone. Second, the researcher asked them if they were familiar with curated app portals. If they were not, DS briefly explained the principle and asked people to repeat the search using the 'NHS Apps Library' and the PHE 'One You Apps' curated health app portals (Figure 2). During the think aloud sessions, positive reinforcement using verbal (e.g. 'You are doing great', 'Right') and non-verbal (e.g. nodding) communication was used to encourage participants to continue to express their views. In quiet moments, prompts were used (e.g. 'What are you thinking now?', 'Tell me what is on your mind'). Following the think aloud task, questions regarding their experience with the uptake of and engagement with apps were asked (see Multimedia Appendix 3 for the topic guide). The sessions lasted between 26 and 63 minutes. Participants received a $£ 20$ gift voucher as compensation for their time.
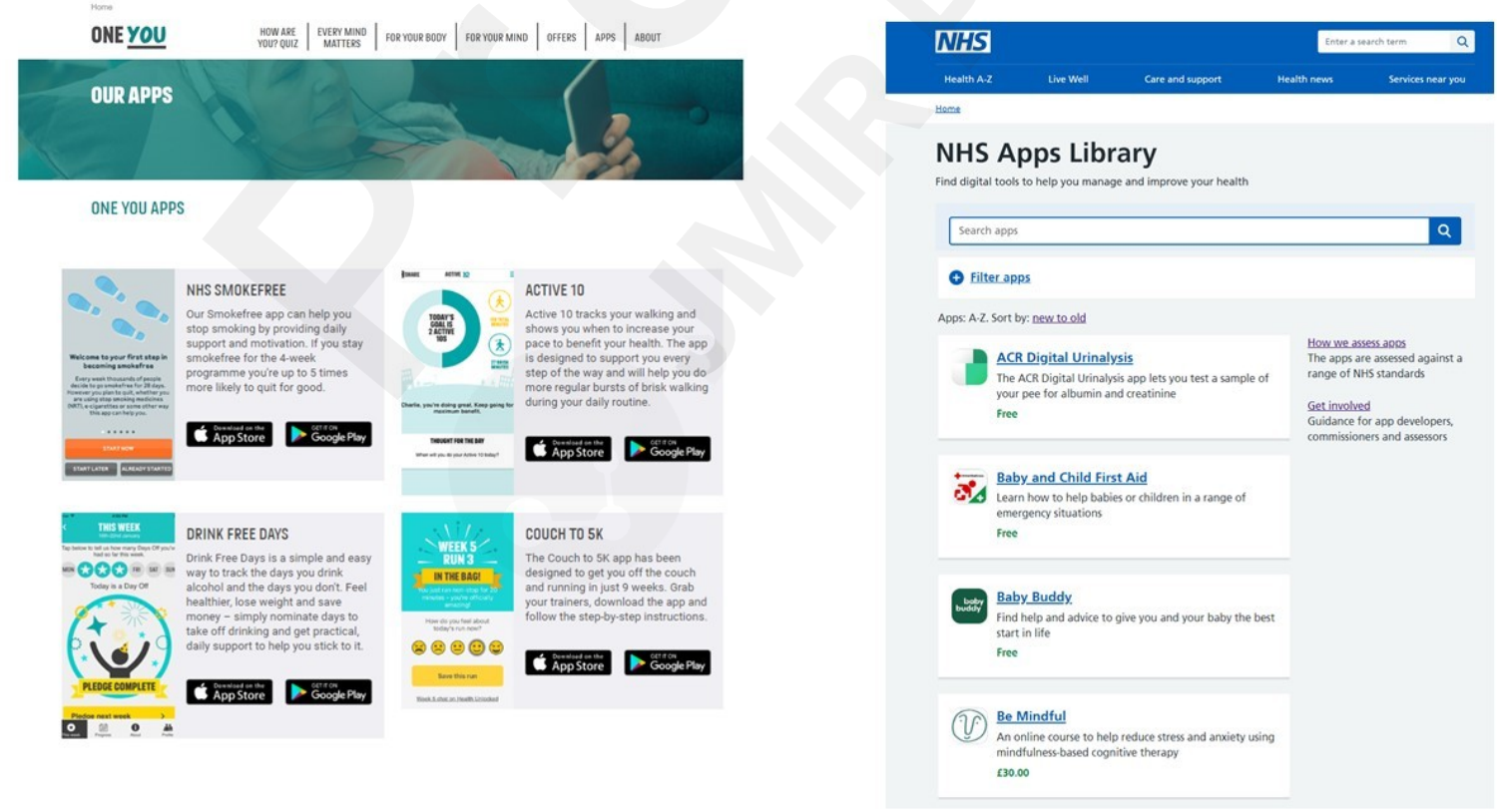

Figure 2. Screenshot of the Public Health England's 'One You Apps' portal and the 'NHS Apps Library'. 


\section{Data analysis}

The sessions were audio-recorded and transcribed verbatim by an external company. Transcriptions were checked for accuracy by the researcher undertaking the interviews. The data were analysed using framework analysis following the stages of familiarisation, identification of thematic framework, indexing, charting, mapping and interpretation [37]. To ensure rigour, trustworthiness and consistency, a percentage of randomly selected transcripts (15\%) were independently coded by the second author (OP). The deductive thematic framework based on the TDF was refined iteratively through repeated discussions with the second author (OP), and any discrepancies were resolved through discussion with the senior author (FN). Indexing was completed by the first author (DS) using QSR NVivo 12. The data were charted, and the responses were grouped according to the finalised thematic framework. During mapping and interpretation, the grouped data were examined by DS to identify patterns. During mapping, identified factors were classified according to their organic position rather than what they affect (e.g. an opportunity factor may indirectly influence the behaviour through increasing the motivation for uptake of a health app, as well as influencing it directly). To aid comprehension of the findings for uptake in general and on health app portals in particular, data were analysed and presented separately for these two topics.

\section{External validity}

To enhance the credibility and trustworthiness of the results [38], six participants (30\%) were randomly selected and requested via email to provide feedback on a document with a summary of the findings and conclusions ('member checking'). They were asked whether they recognised their opinions and whether they agreed with the interpretation of the findings. Two participants responded to our request and confirmed that their opinions had been captured. In one case, our email failed to be delivered.

\section{Reflexivity}

The researchers involved in this study are mixed-methods researchers with experience applying the COM-B model and the TDF to qualitative data. She disclosed her research interest to participants on the day of the interview and no prior relationship was established between her and participants. The interviews were conducted by the lead author, a PhD candidate who has undertaken extensive training in the collection and analysis of qualitative data. Participants were encouraged to share their thoughts (both positive or negative) and to be honest. The interviewer felt that good rapport was built with the interviewees, and most participants $(n=16)$ expressed their interest in learning more about the findings of the research. Field notes and a research journal was kept during data collection.

\section{Results}

A total of 18 participants completed the interview. The average age of participants was 43 years $(S D=14), 9(50 \%)$ were females, $14(78 \%)$ were of white British ethnicity, (13) 72\% were employed full time, 2 (11\%) had postgraduate qualifications, $17(94 \%)$ had used health apps before, and $11(61 \%)$ were using health apps at the time of the interviews, out of which $8(73 \%)$ reported daily health app usage. Most participants were interested in changing more than one behaviour (e.g. losing weight, getting more active, managing mood) and only $16 \%$ of participants expressed a desire to change only one behaviour. Participants' characteristics are presented in Multimedia Appendix 3.

Two participants were satisfied with the app they were already using and did not wish to take part in the think aloud exercise to look for a different app. The remaining 16 participants searched for apps targeting physical activity ( $\mathrm{n}=6$ ), weight management $(n=4)$, mood and mental wellbeing $(n=3)$, smoking cessation $(n=1)$, alcohol reduction $(n=1)$ and sleep $(n=1)$.

The findings pertaining to factors relevant for both the uptake of health apps and views on curated health app portals are presented under the components of the COM-B model: Capability, Opportunity, Motivation. Higher order themes and subthemes informed by the COM-B model and the TDF are reported in Table 1.

Table 1. Factors influencing uptake of health apps in general and on health app portals mapped onto the components of the COM-B model and TDF constructs.

\begin{tabular}{|l|l|l|l|}
\hline $\begin{array}{l}\text { COM-B } \\
\text { and TDF }{ }^{\text {b }} \text { construct }\end{array}$ & Identified factor & Uptake in general & Uptake on health app portals \\
\hline Physical Capability & App literacy & $\bullet$ Technological competency & - \\
\hline & Skills & & \\
\hline \multicolumn{2}{l}{ Psychological Capability } \\
\hline
\end{tabular}




\begin{tabular}{|c|c|c|c|}
\hline \multirow[t]{4}{*}{\begin{tabular}{l|l} 
Knowledge \\
\end{tabular}} & Health awareness & $\begin{array}{l}\text { - General health consciousness or } \\
\text { having family members } \\
\text { diagnosed with a condition or } \\
\text { disease, or concerns regarding a } \\
\text { behaviour or health outcome }\end{array}$ & - \\
\hline & App awareness & $\begin{array}{l}\text { Knowledge of the existence of } \\
\text { health and wellbeing apps }\end{array}$ & $\begin{array}{l}\text { - Knowledge of the existence of } \\
\text { health and wellbeing apps listed on } \\
\text { health app portals }\end{array}$ \\
\hline & User guidance & - & $\begin{array}{l}\text { - Instructions on how to effectively } \\
\text { use a health app portal }\end{array}$ \\
\hline & Health information & - & $\begin{array}{l}\text { - Educational information related to } \\
\text { health and wellbeing }\end{array}$ \\
\hline \begin{tabular}{|l|} 
Memory, \\
attention, \\
decision \\
processes
\end{tabular} & Cognitive load & - & $\begin{array}{l}\text { - The manner in which apps are } \\
\text { presented on the portal } \\
\text { - The complexity of the search or to } \\
\text { access a relevant health app }\end{array}$ \\
\hline \multicolumn{4}{|l|}{ Physical Opportunity } \\
\hline \multirow[t]{4}{*}{$\begin{array}{l}\text { Environmental } \\
\text { resources }\end{array}$} & Availability & $\begin{array}{l}\text { - The ability to use a smartphone } \\
\text { anytime, anywhere } \\
\text { - Availability of an app on all major } \\
\text { commercial app stores }\end{array}$ & \\
\hline & $\begin{array}{l}\text { Portal tailored to } \\
\text { individuals needs }\end{array}$ & - & $\begin{array}{l}\text { - Personalised listing of apps } \\
\text { targeting age, gender, health } \\
\text { condition }\end{array}$ \\
\hline & Cost of an app & $\begin{array}{l}\text { - Low cost and apps that are free } \\
\text { for users }\end{array}$ & $\begin{array}{l}\text { - Low cost and apps that are free } \\
\text { for users }\end{array}$ \\
\hline & Aesthetics & - The look and design of an app & $\begin{array}{l}\text { - User-friendly and design related } \\
\text { characteristics of the portal }\end{array}$ \\
\hline \multicolumn{4}{|l|}{ Social Opportunity } \\
\hline $\begin{array}{l}\text { Social } \\
\text { influences }\end{array}$ & Social influences & $\begin{array}{l}\text { - The importance of reviews and } \\
\text { ratings in the commercial app } \\
\text { stores, as well as of apps } \\
\text { promoted as 'editor's choice' } \\
\text { - Identified credible sources: apps } \\
\text { developed or endorsed by } \\
\text { trusted app developers, } \\
\text { organisations, universities, or } \\
\text { promoted by respected } \\
\text { celebrities (e.g. athletes) } \\
\text { - Recommendations received } \\
\text { from health practitioners or from } \\
\text { friends and family }\end{array}$ & $\begin{array}{l}\text { - Health app portals perceived as } \\
\text { credible source } \\
\text { - Recommendations of health app } \\
\text { portals needed mainly in primary } \\
\text { care } \\
\text { - Clarity about the recommended } \\
\text { apps on health app portals } \\
\text { - Explanations about any required } \\
\text { GP referral }\end{array}$ \\
\hline \multicolumn{4}{|l|}{ Reflective Motivation } \\
\hline \multirow{4}{*}{$\begin{array}{l}\text { Beliefs about } \\
\text { consequences }\end{array}$} & $\begin{array}{l}\text { Perceived } \\
\text { competence }\end{array}$ & $\begin{array}{l}\text { - App preferred over face-to-face } \\
\text { intervention when the user feels } \\
\text { that they can engage with the } \\
\text { app on their own }\end{array}$ & - \\
\hline & Time efficiency & $\begin{array}{l}\text { - The ability of a health app to be } \\
\text { interacted with a minimum } \\
\text { amount of time }\end{array}$ & - \\
\hline & $\begin{array}{l}\text { The perceived } \\
\text { utility of the app }\end{array}$ & $\begin{array}{l}\text { - Discrepancy between what the } \\
\text { users are looking for and what } \\
\text { the app offers, characterised by a } \\
\text { relevant title, description, } \\
\text { pictures, adaptation to individual } \\
\text { characteristics and users' } \\
\text { previous experience with health } \\
\text { apps }\end{array}$ & $\begin{array}{l}\text { - Discrepancy between what the } \\
\text { users are looking for and what the } \\
\text { app listed on health app portal } \\
\text { offers, characterised by a relevant } \\
\text { title, description, pictures }\end{array}$ \\
\hline & $\begin{array}{l}\text { Perceived } \\
\text { accuracy }\end{array}$ & $\begin{array}{l}\text { The perceived effectiveness of } \\
\text { apps before the selection of an } \\
\text { app }\end{array}$ & $\begin{array}{l}\text { - Potential app users' perceived } \\
\text { effectiveness of apps listed on } \\
\text { health app portals }\end{array}$ \\
\hline
\end{tabular}




\begin{tabular}{|c|c|c|c|c|}
\hline & & Data protection & $\begin{array}{l}\text { - Concern regarding the handling } \\
\text { of personal data }\end{array}$ & $\begin{array}{l}\text { - Concern over the handling of } \\
\text { personal data }\end{array}$ \\
\hline & Intentions & Commitment & $\begin{array}{l}\text { The level of commitment when } \\
\text { deciding to download a health } \\
\text { app }\end{array}$ & - \\
\hline & Social identity & Social identity & $\begin{array}{l}\text { - Identity related to app use (e.g. } \\
\text { trends and gender specificity) }\end{array}$ & $\begin{array}{l}\text { - Identity related to app use (e.g. } \\
\text { feeling like a 'patient') }\end{array}$ \\
\hline \multicolumn{5}{|c|}{ Automatic Motivation } \\
\hline & \multirow[t]{3}{*}{ Emotions } & Positive & $\begin{array}{l}\text { Triggered by curiosity in trying a } \\
\text { health app, and by the time- } \\
\text { efficiency characteristic of an app } \\
\text { as opposed to face-to-face } \\
\text { interventions, as well as by being } \\
\text { provided by a credible source }\end{array}$ & $\begin{array}{l}\text { - Triggered by curiosity in choosing } \\
\text { a behaviour change tool from a } \\
\text { curated health app portal, and from } \\
\text { a credible source }\end{array}$ \\
\hline & & $\begin{array}{l}\text { Negative } \\
\text { emotions }\end{array}$ & $\begin{array}{l}\text { - Triggered by lack of availability } \\
\text { on all major app stores } \\
\text { - Preferred over a face-to-face } \\
\text { intervention if feeling anxiety } \\
\text { (e.g. caused by an unhealthy } \\
\text { behaviour or unhealthy state), } \\
\text { and pressure (to succeed or to } \\
\text { show progress) }\end{array}$ & $\begin{array}{l}\text { - Triggered by lack of search } \\
\text { features on the portal, or when the } \\
\text { search yields irrelevant results; } \\
\text { when an app requires GP referral } \\
\text { without further explanation, when } \\
\text { an app is only available in one } \\
\text { major app store }\end{array}$ \\
\hline & & Mixed emotions & $\begin{array}{l}\text { Triggered by the aesthetics } \\
\text { (design) of the apps and by } \\
\text { adaptation to individual } \\
\text { characteristics (judged by the } \\
\text { title, description, pictures, gender } \\
\text { specificity) }\end{array}$ & $\begin{array}{l}\text { - Triggered by the aesthetics and } \\
\text { features of the portal and the } \\
\text { perceived utility of the apps }\end{array}$ \\
\hline
\end{tabular}

${ }^{a}$ COM-B: capability, opportunity, motivation, behaviour model.

'TDF: theoretical domains framework.

\section{Factors influencing the uptake of health and wellbeing apps}

Half of participants who agreed to search for a health app $(n=8)$ used Google Search as their first choice to find a suitable app, while the other half opened a commercial app store. The latter search among hundreds of available apps was described by most participants as difficult or a "minefield" (P2, P4, P6). One participant described this task as being "far more complicated than I thought it would be" (P2). By the end of this exercise, only three participants found an app that they were willing to download and engage with further to change their behaviour.

\section{Capability factors related to the uptake of health and wellbeing apps in general}

Participants who presented a higher level of technological competency were able to better navigate on their phones, thus highlighting that app literacy skills are necessary when selecting a health app. One participant, who had never used a health app before, showed signs of technical difficulties (i.e. lack of skills) during the think aloud exercise while searching for an alcohol reduction app in a commercial app store.

"I wouldn't know how to do that [refining the search to find a suitable app]." (P12)

Additionally, two participants expressed their concern toward the older generation and stated that training should be provided for those with insufficient technological and app literacy skills.

"My nanny is diabetic and if there was an app to help her with her diabetes, then I'm sure she would be happy to use it but it's just someone would need to explain it to her." (P18)

All participants expressed their decision to look for an app for health reasons, such as getting healthier or to prevent illness. This included reasons of being diagnosed, or having a family member diagnosed, with a medical condition (e.g. diabetes, high blood pressure), concern of the negative effect a current behaviour may have (e.g. smoking, alcohol consumption), to better manage or improve their mental health (e.g. anxiety, self-confidence) and general wellbeing (e.g. sleep quality):

"I'm trying to avoid having type 2 diabetes, or getting it, so there's a background, my mother, in my family, there's a heart conditions background, which is why l'm really wanting to do something about my health." (P3)

While most participants were aware of the existence of some apps, three participants were surprised by the existence of health apps for smoking cessation and mental health issues.

"It didn't cross my mind that I could use an app for stopping smoking, so it is new." (P16) 


\section{Opportunity factors related to the uptake of health and wellbeing apps in general}

Some participants expressed their preference to look for a health app as a digital behaviour change intervention instead of a face-to-face intervention because of the availability and the low cost of an app. However, concerns around widening inequalities were raised by one participant who showed signs of worry about the limited access to digital aids for individuals living in deprived areas.

"So if they [people living in deprived areas] do not have the smart phone, they won't be able to use it, so it's not going to work, is it? It's what happened with the Universal Credit, so it's not going to work. I mean issue everyone a smart phone." (P16)

A few participants highlighted the importance of the availability of health apps in both major commercial app stores (Apple App Store and Google Play), not just one or the other.

Most participants stated that apps should be available at no cost. Only six participants expressed their willingness to pay a small fee for an app if, for example, it would be "almost life-changing" (P4) or if it would include online professional support.

The specific design and colour scheme preferred by participants appeared to be unique and dependent on the individual's taste. However, the majority were looking for a "simple" looking app.

Social influences appeared to be one of the core factors that shaped the selection of apps for all participants during the think aloud exercise. This includes ratings and reviews of the app, how credible the source of the app is and recommendations of apps received from others. Within app stores, most participants described looking at the star ratings and the number of downloads of each app, and whether the apps were listed as an 'editor's choice'. Three participants acknowledged that reviews were subjective, they still reported feeling influenced by the ratings of the app. Additionally, two participants reported that they were sceptical of the reviews, which they believed may have been paid for, and that reviews are not enough, as more information is necessary to make an informed choice.

"You know, so you're having to make all these judgements about people's reviews and then you know deep down that the reviews might be paid for and, you know, it's a bit of a minefield which is why I would only take a free sample and then see if it works for me." (P6)

A credible source was also important. Apps developed or recommended by trusted organisations or respected celebrities seemed more appealing to all participants. Participants who used Google Search to find an app aimed to look for websites they were familiar with or had used before, or for websites that would post 'Top 10 apps for ...' type of articles. Additionally, word of mouth was another identified source of social influence for many.

"I see two different specialists, I have a lung problem as well and I see a lung specialist at a hospital near me and she said to me, the best thing that I could do, which was downloading the Couch to $5 \mathrm{k}$ app." (P14)

\section{Motivational factors related to the uptake of health and well being apps in general}

Health or well-being apps were preferred over face-to-face options because participants reported feeling competent changing their behaviour through the use of an app, requiring less time commitment and avoiding the anxiety and pressure of interacting with others. Time appeared to be a particularly valuable resource for all participants, and they believed apps to have this advantage.

Another core factor in the selection of an app was the way users perceived its utility. This was found to be based on two aspects. First, they appeared to judge how the app is adapted to the individual by reading the title and the description of the app, and by looking at pictures (i.e. screenshots). Twelve participants reported the need for enough information about an app to make an informed choice.

"I would definitely judge more from the pictures more than anything and I think that just nowadays everyone does, is you get an idea of the app from the pictures. (...) I mean I think when you see an older person on a picture and you're a lot younger, it makes you think, I mean it's the wrong think to think but it makes you think maybe it's not for me." (P7)

Second, it seemed that twelve participants relied on their past experiences with health apps. Whether those experiences were positive or negative may have shaped their beliefs about health apps in general.

"So that's why My Fitness Pal is the first app that I've ever had that's actually worked." (P9)

Additionally, seven participants expressed their scepticism about the accuracy and effectiveness of some apps (e.g. mental health apps), and concerns about data protection were mixed.

“These mindful ones, I've never downloaded one and l'm sceptical." (P17)

Participants mentioned that commitment to the behaviour change would influence uptake and future engagement. 
"So I think the committed ones seek out the ones that are the right ones for them, the best ones, rather than necessarily the trendy ones." (P4)

Participants' social identity also shaped their selections. Many reported that they did not wish to select apps that promoted groups they do not seem themselves fitting in with (e.g. athletic body image or individuals of the hipster subculture).

“They've got a kind of hipster bloke and now they've got a kind of sexy female image with tattoos down her arm, sexy, trendy, female image. Okay, so they are obviously aiming at younger, sort of people in their twenties and thirties, yeah, another sexy female image. It's quite interesting isn't it, I'm looking at the images and not the words and getting a sense, is this for me, middle aged, well older woman?!" (P6)

Curiosity, defined here as a desire to learn something, was the only stand-alone positive emotion, and appeared to positively influence the uptake of health apps for many participants.

"I thought out of curiosity l'd have a look, so I just typed in quit smoking in Google play store and there's hundreds of apps from various people with varying degrees of credibility, and they all were pretty similar to be honest." (P13)

Apps linked to a credible source were important with people unimpressed when an app was not available on all major app stores.

\section{Views on curated health app portals}

None of the participants spontaneously used a curated portal. Curated portals were then introduced to the participants but none were previously aware of them. Curated health app portals were appealing to all participants and they believed the portals would be likely to engender trust. However, searching for a health app on NHS Apps Library and the One You App portal was a generally disappointing experience. Only two participants chose a health app from a health app portal (One You Apps), while the rest of the participants decided to continue the search in commercial app stores.

\section{Capability factors related to the uptake of health and wellbeing apps on health portals}

All participants had heard of widely advertised apps (e.g. Couch to 5k), but none were aware before study participation of the existence of curated health app portals.

"I think they're brilliant [apps on health app portals]; I didn't know they existed." (P11)

Navigating on the NHS Apps Library seemed easy for some. However, a few participants mentioned that a user guide or help section would be a useful added feature of the portal. Two participants reported that they did not find it easy to use the filter features, and in many cases, they felt the search yielded irrelevant results (e.g. while searching for a physical activity app the results also listed apps for mental health). A few participants reported that navigating on curated app portals felt difficult, characterised as "cumbersome" (P4, P12).

"It's not clear, it's suggests that they are independent apps but maybe they should have some guidelines about design, you know, of their sort of landing pages." (P6)

\section{Opportunity factors related to uptake on health portals}

All participants indicated that they would want a portal tailored to their needs with categories related to their gender, age group, and medical conditions they may have.

"So something like that, this is suitable if you're over 65, this would be more suitable for you if you're under 40 or with these ones that you don't have to go and see your GP, that you can pay for, if you have any concerns, visit your GP or speak to a health professional because some people don't have that common sense." (P14)

Participants had different opinions about the layout of these portals. Some liked the NHS Apps Library design better, with simple colours, while others enjoyed the more colourful One You App portal. Most participants felt that a fusion between these two designs (the searchability and filters of NHS Apps Library and the look and presentation of the One You App portal) and a better functionality would create the ideal curated health app portal. "Why they are not combined?" (P8)

While many participants expressed their wish to access apps for free, a few participants were more open to pay for an app that was listed on a curated health app portal.

"This is fabulous, and l'd be much more inclined to pay money. This is really, really good." (P6)

Participants found the NHS and PHE trustworthy and believed these portals would provide safe and effective digital aids. Some indicated a desire to receive further recommendations for using this portal from their primary care physicians.

"If GPs knew that they could say 'well this could help you' I'm sure that they would recommend it to people." (P11)

However, they also wanted to avoid putting unnecessary pressure on GP practices.

"You've got "free but requires GP referral" and when you're thinking the NHS is under so much financial strain and pressure at the moment, why do I need a GP referral to obtain an app?" (P2) 
Additionally, the One You App portal lists a few apps that are recommended, but participants expressed their confusion and lack of clarity of why some apps are 'recommended', and by whom.

\section{Motivation factors related to uptake on health portals}

While searching on curated health app portals none of the participants expressed signs of concern about data protection and accuracy of apps, although two participants reported that they would want to read more about how these apps were developed and tested.

"How long it takes, how many sessions and the fact that it's been tested in clinical trials and evaluated by NICE which, to me, is probably quite an important thing." (P1)

Social identity was also important. Some participants had identified themselves as individuals living with a medical condition. These participants were keen to look for an app that targets the behaviour change of individuals with preexisting medical conditions. Others stated that they do not wish to feel "like a patient" (P7) and seemed reluctant to continue the search on a curated health app portal.

"So it would be nice to have one specific for maybe people with medical problems or age-related problems, etc." (P15)

\section{Discussion}

\section{Principal Findings}

The online search for health and wellbeing apps was found to be difficult. Factors influencing uptake of health apps were mapped under the COM-B model and the Theoretical Domains Framework (TDF). We found that social influences and participants' beliefs about consequences (the perceived utility of the app) are key factors influencing the uptake of health apps. This conclusion was based on the frequency and salience of themes as these occurred during the interview. Curated health portals were found appealing to all despite of the lack of awareness of their existence. However, the way apps are currently presented on these portals did not meet user's needs due to a lack of certain features, such as lack of tailoring to the user's requirements.

In line with previous research, the findings revealed the importance of the capability and opportunity factors, such as app literacy skills, health awareness and app awareness, the aesthetics of the app, the low cost of an app, reading reviews and checking ratings, the credible source, and recommendations of apps from others including health professionals [18, $22,39,40]$. Interestingly, the perception of the cost of an app appeared to be related to the perceived utility and the credibility of the source. Although at the start, some participants were against paying for apps, the more useful an app was perceived, the more inclined participants felt to pay a fee. This phenomenon was observed for apps listed on health app portals which were considered a credible source. More importantly, unlike apps listed on commercial app stores, there was implied trust in apps listed on curated health app portals by participants. Additionally, some health apps are not available to download in both commercial app stores. Participants found it disappointing that some apps were only available for iPhone users. This is in line with previous research which found that out of eighteen investigated health apps, only one third were available to download on both major commercial app stores [28].

In terms of motivational factors, we found that the perceived utility included aspects related to the individuals' perceptions about the presentation of an app as well as their previous experiences with health apps. Together these shaped the way participants judged the usefulness of an app. This characterisation underlines the need expressed by others previously for a better way to present health apps through a description that would lead to an informed choice (e.g. the content of the app) [25-27], and potentially positively affect other motivational factors, such as the accuracy of an app and data protection [41]. Notably, concern about data protection and the accuracy of a health app was minimal when participants navigated on health app portals as opposed to commercial app stores.

There is a need to understand what design aspects generate positive or negative emotions, and for whom. Emotions are powerful driver of a behaviour, which affect decision making (e.g. app uptake) [42]. A key emotion identified in this research directly influencing uptake was curiosity. However, this study emphasised the importance of positive emotions triggered by, for example, the credible source of an app, and negative emotions triggered by restriction of information (e.g. lack of understanding of the necessity of GP referral to download an app). Taking these into consideration may lead to better uptake with such tools.

Uptake and engagement are connected. Engagement without uptake is not possible, and uptake without taking into consideration factors that are important for engagement is impractical. Some factors might influence both uptake and engagement; for example, our research suggests that the perceived utility of an app is one of the main factors for uptake. However, a previous study found that perceived utility was a predictor for engagement with an alcohol reduction app [43]. Hence, where possible, uptake and engagement should be considered together as two linked constructs.

\section{Strengths and Limitations}

The main strength of this research lies in the methodology. Given the aim of this study was to explore uptake with health apps and by applying a user-centred approach, the think aloud methodology was the appropriate technique to use [33, 44] as it will minimise recall bias. Involving stakeholders, patient and public engagement representatives and policy makers, in 
the design of the research enhances scientific rigour. The purposive sampling technique adopted enabled the recruitment of a wide range of participants that included the same number of females and males and having different levels of education and employment status, and the sample overrepresented ethnicity relative to local rates. Having used the COM$\mathrm{B}$ and the TDF theoretical frameworks to guide the data analysis is another strength of this research.

The research has several limitations. First, asking participants to perform the think aloud task under observation may not be fully analogous to how they would perform a search when on their own. Second, some identified factors were difficult to define and describe due to lack of specificity of the description provided by participants. These include aesthetics of apps, often described vaguely ('nice', 'elegant') and the cognitive load associated with engagement with these ('easy to use'). Third, for a qualitative research study exploring such a broad topic, we felt information saturation was reached, but it is possible that additional participants with more varied characteristics would have allowed us to identify additional concepts. Finally, during external validation a randomly selected subsample of participants was asked via email to provide feedback on the summary of findings. Three participants (50\%) did not reply, and it is unclear whether these participants ignored our request or did not agree with the interpretation of the results.

\section{Implications for research, policy and practice}

This research has important implications for stakeholders in public health and policymakers that target prevention and health promotion using digital technologies, and governmental bodies and trusted health organisations that provide curated health app portals. Low awareness, low app literacy skills, lack of availability on all major app stores, and lack of recommendation in primary care were identified as factors limiting the uptake of health apps in general and on curated app portals. These are factors are important to consider for improving the uptake of health apps. The selection was described as difficult. Hence, there is a need for public guidance on how to identify evidence-based tools [18, 22], and for health practitioners to promote and advise their patients on how to select appropriate health and wellbeing apps [40]. Raising awareness of such tools through both online and offline promotion channels might provide better access to effective apps.

Our findings could also help developers reconsider the ways in which apps are currently presented on commercial app stores and app portals, which might in turn increase the uptake of evidence-informed health apps. The idea of selecting an app from a health app portal was appealing to all participants, although individuals' needs were not currently met. These findings describe essential barriers and facilitators related to participants' capability, opportunity and motivation to take up health and wellbeing apps. For example, app descriptions and presentations that better align with individuals' needs may increase the uptake of health apps on health app portals. These findings can also be used to inform the development of interventions that specifically aim to promote the uptake of and engagement with evidence-informed health and wellbeing apps, a priority within the NHS Long Term Plan (i.e. 'digital first'). By targeting the identified psychological influences on app uptake through further interventional work, organisations that provide app portals (e.g. the NHS, PHE) should be able to increase their impact through helping people to better select appropriate apps. A summary of recommendations for policy makers, providers and developers is presented in Table 2.

Table 2. Recommendations for policy makers, industry, health care providers and app developers for a better uptake of health and wellbeing apps.

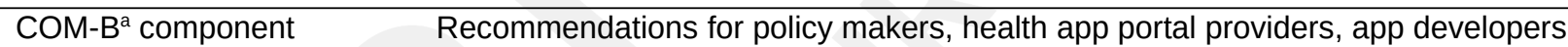

1. Capability

2. Opportunity
1.1. Improve app literacy skills with a focus on elderly and marginalised populations and continue working towards reducing the digital divide (e.g. through the use of an outreach approach to target elderly, migrant and homeless populations)

1.2. Increase awareness of effective health apps and curated health app portals through promotion online and offline in primary care, mass media and public spaces

1.3. Provide guidance on how to use a health app portal (e.g. through incorporating an extensive help section) and additional physical and mental health related evidence-based articles

1.4. Promote reduced cognitive load on curated health app portals (e.g. through the use of images and short app descriptions)

2.1. Ensure evidence-informed apps are available for free or at low cost to everyone

2.2. Make apps available on all major app stores simultaneously

2.3. Offer the possibility to tailor the health app portal to target certain demographics (e.g. apps for physical activity for women aged 60 and over)

2.4. Offer apps at low cost and provide explanation for those that require referrals and justifications for the cost of paid apps on curated health app portals

2.5. Collaborate with interaction design experts and end-users to enhance the aesthetics of health app portals

2.6. Promote evidence-informed apps via trusted organisations and provide information on how the apps were developed and tested 
3. Motivation
2.7. Encourage health professionals and practitioners of promotion of evidenceinformed health apps and health app portals

3.1. Provide relevant and realistic titles and avoid general app descriptions. Descriptions should be short, but contain details of what the app offers and how it is able to help the user

3.2. Provide pictures of the app (e.g. screenshots) and avoid pictures that promote an unrealistic body image

3.3. Provide information about the accuracy and effectiveness of the app (e.g. details about development and developers), as well as about how the users' data are handled

3.4. Take into account the user's emotions about certain features by constantly involving users in the development of health apps

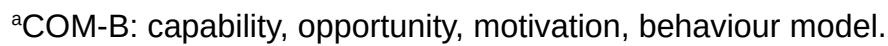

\section{Future research}

Future research is needed to minimise factors limiting uptake, such as low awareness, low app literacy skills and a lack of recommendation in primary care. Our results suggest that there is a need to better tailor the design and content of health app portals to better meet individuals' needs. However, the mixed views on specific app designs indicates that more research is needed to investigate whether there are general design principles that are missed and could be followed to accommodate the majority of people, or whether better tailoring and/or adaptive interventions should be considered instead. Future research may also want to consider comparing curated health app portals developed by private organisations with those developed by governmental bodies to investigate whether portal design related features are considered less or more important than credibility and trust in apps listed on them. Experimental research is needed to assess whether there is a trade-off between credibility, social influences and perceived utility of the apps presented on curated health app portals. Furthermore, with a growing concern around widening inequalities [45], solutions should be focused on reducing the digital divide and health inequalities that may appear as a result of financial constraint of owning a smartphone and lack of sufficient app literacy skills.

\section{Conclusion}

Among factors mapped under capability, opportunity and motivation components of the COM-B model, social influences and the perceived utility of an app appear to be the core factors influencing uptake in general and on curated health app portals. Curated app portals are considered trustworthy and serve as a credible source for apps, however there is disappointment with their current implementation. Uptake on health app portals, as opposed to uptake in general, appears to help address people's concerns regarding data protection and accuracy of apps. Health organisations that develop app portals may consider targeting the factors identified across the COM-B and the TDF as part of additional experimental work as this could help to increase impact through better selection of appropriate health apps.

\section{Acknowledgments}

We would like to thank to all participants for their contribution to the study. The authors would like to acknowledge the support on the development of topic guide received from RP and SH (patient and public representatives) the Deputy Director of PHE Digital, the PHE Strategy and Planning and PHE Strategy and Innovation lead.. Furthermore, the authors are grateful to the University College London Tobacco and Alcohol Research Group for their expert opinion on the data analysis. DS is funded through a Doctor of Philosophy studentship, provided jointly by PHE and the University of East Anglia (R205853HSC). OP receives salary support from Cancer Research UK (C1417/A22962).

\section{Authors' Contributions}

DS, FN, AJ, TC and JB conceptualised the study design. DS wrote the study protocol with contributions from FN, AJ, TC, JB. All authors commented on the topic guide. DS undertook recruitment of participants, data collection, data analysis, interpretation and report writing. OP double coded a proportion of the transcripts. DS, OP and FN finalised the final thematic framework. DS prepared the manuscript. All authors read, commented and contributed on the final manuscript.

\section{Conflicts of Interest}

$\mathrm{JB}$ has received unrestricted research funding to study smoking cessation from pharmaceutical companies who manufacture smoking cessation medications. JB, FN, OP and DS are unpaid members of the scientific committee for the 'Smoke Free' app and have no financial interest in the app. 


\section{Abbreviations}

COM-B model: Capability, Opportunity, Motivation - Behaviour model COREQ: Consolidated Criteria of Reporting Qualitative Research

GP: General Practitioner

NHS: National Health Service

PHE: Public Health England

SD: Standard deviation

TDF: Theoretical Domains Framework

UK: United Kingdom

Multimedia Appendix 1: COREQ checklist

Multimedia Appendix 2: Screening questionnaire

Multimedia Appendix 3: Topic guide for the interviews

Multimedia Appendix 4: Study participants characteristics 


\section{References}

1. World Health Organisation. Ten Threats to Global Health in 2019. 2019 [01.02.2020]. Available from: https://www.who.int/news-room/spotlight/ten-threats-to-global-health-in-2019.

2. World Health Organisation. World Health Organistion Priorities: Health for all2020 01.06.2020. Available from: https://www.who.int/dg/priorities/health-for-all/en/.

3. National Health Service (NHS). The NHS long term plan2019. Available from: https://www.longtermplan.nhs.uk/.

4. Public Health England. Public Health England Startegy 2020-25. Executive summary [Internet]. 2019 01.02.2020. Available from: https://assets.publishing.service.gov.uk/government/uploads/system/uploads/ attachment_data/file/830105/PHE_Strategy_2020-25_Executive_Summary.pdf.

5. Coughlin SS, Whitehead M, Sheats JQ, Mastromonico J, Smith S. A Review of Smartphone Applications for Promoting Physical Activity. Jacobs journal of community medicine. 2016;2(1):021. PMID:27034992

6. Coughlin SS, Jacobs M, Thind H, Champagne N, Liu B, Golden MS, et al. On the Need for ResearchTested Smartphone Applications for Reducing Exposures to Known or Suspected Breast Carcinogens in Work and Home Environments. Journal of environment and health sciences. PMID:26688831 2015;1(4):10.15436/2378-6841.15.e004.

7. Zhao J, Freeman B, Li M. Can Mobile Phone Apps Influence People’s Health Behavior Change? An Evidence Review. Journal of Medical Internet Research. 2016;18(11):e287. PMID:27806926

8. Klasnja P, Pratt W. Healthcare in the pocket: mapping the space of mobile-phone health interventions. Journal of biomedical informatics. 2012;45(1):184-98. PMID:21925288

9. Whittaker R, McRobbie H, Bullen C, Rodgers A, Gu Y. Mobile phone-based interventions for smoking cessation. Cochrane Database of Systematic Reviews. 2016(4). PMID: 27060875

10. Flores Mateo G, Granado-Font E, Ferre-Grau C, Montana-Carreras X. Mobile Phone Apps to Promote Weight Loss and Increase Physical Activity: A Systematic Review and Meta-Analysis. J Med Internet Res. 2015;17(11):e253. PMID:26554314

11. Schoeppe S, Alley S, Van Lippevelde W, Bray NA, Williams SL, Duncan MJ, et al. Efficacy of interventions that use apps to improve diet, physical activity and sedentary behaviour: a systematic review. International Journal of Behavioral Nutrition \& Physical Activity. 2016;13(1):127. PMID:27927218

12. Nour M, Chen J, Allman-Farinelli M. Efficacy and External Validity of Electronic and Mobile PhoneBased Interventions Promoting Vegetable Intake in Young Adults: Systematic Review and Meta-Analysis. Journal of Medical Internet Research. 2016;18(4):e58. PMID:27059765

13. Lee S, Lindquist R. A review of technology-based interventions to maintain weight loss. Telemedicine Journal \& E-Health. 2015;21(3):217-32.

14. Sherifali D, Nerenberg KA, Wilson S, Semeniuk K, Ali MU, Redman LM, et al. The Effectiveness of eHealth Technologies on Weight Management in Pregnant and Postpartum Women: Systematic Review and Meta-Analysis. Journal of Medical Internet Research. 2017;19(10):e337. PMID:29030327

15. Choo CC, Burton AAD. Mobile Phone Apps for Behavioral Interventions for At-Risk Drinkers in Australia: Literature Review. JMIR Mhealth Uhealth. 2018;6(2):e18. PMID: 29439946

16. Husain I, Spence D. Can healthy people benefit from health apps? BMJ : British Medical Journal. 2015;350:h1887. PMID:25873345

17. Weisel KK, Fuhrmann LM, Berking M, Baumeister H, Cuijpers P, Ebert DD. Standalone smartphone apps for mental health—a systematic review and meta-analysis. npj Digital Medicine. 2019;2(1):118. PMID:31815193

18. Szinay D, Jones A, Chadborn T, Brown J, Naughton F. Influences on the Uptake of and Engagement With Health and Well-Being Smartphone Apps: Systematic Review. J Med Internet Res. 2020. PMID:32348255

19. Krebs P, Duncan DT. Health App Use Among US Mobile Phone Owners: A National Survey. JMIR Mhealth Uhealth. 2015;3(4):e101. PMID:26537656

20. Crane D, Garnett C, Brown J, West R, Michie S. Behavior change techniques in popular alcohol reduction apps: content analysis. Journal of medical Internet research. 2015;17(5):e118. PMID:25977135 21. Wyatt JC. How can clinicians, specialty societies and others evaluate and improve the quality of apps 
for patient use? BMC Medicine. 2018;16(1):225. PMID:30501638

22. Donker T, Petrie K, Proudfoot J, Clarke J, Birch M-R, Christensen H. Smartphones for Smarter

Delivery of Mental Health Programs: A Systematic Review. J Med Internet Res. 2013;15(11):e247. PMID:24240579

23. Radovic A, Vona PL, Santostefano AM, Ciaravino S, Miller E, Stein BD. Smartphone Applications for Mental Health. Cyberpsychol Behav Soc Netw. 2016;19(7):465-70. PMID:27428034

24. Terhorst Y, Rathner EM, Baumeister H, Sander L. «Hilfe aus dem App-Store?»: Eine systematische Übersichtsarbeit und Evaluation von Apps zur Anwendung bei Depressionen. Verhaltenstherapie. 2018;28(2):101-12.

25. Albrecht U-V, Malinka C, Long S, Raupach T, Hasenfuß G, von Jan U. Quality Principles of App Description Texts and Their Significance in Deciding to Use Health Apps as Assessed by Medical Students: Survey Study. JMIR Mhealth Uhealth. 2019;7(2):e13375. PMID: 30810534

26. Wykes T, Schueller S. Why Reviewing Apps Is Not Enough: Transparency for Trust (T4T) Principles of Responsible Health App Marketplaces. J Med Internet Res. 2019;21(5):e12390. PMID:31045497

27. Charbonneau DH, Hightower S, Katz A, Zhang K, Abrams J, Senft N, et al. Smartphone apps for cancer: A content analysis of the digital health marketplace. Digit Health. 2020;6:2055207620905413. PMID:32110428

28. Baxter C, Carroll J-A, Keogh B, Vandelanotte C. Assessment of Mobile Health Apps Using Built-In Smartphone Sensors for Diagnosis and Treatment: Systematic Survey of Apps Listed in International Curated Health App Libraries. JMIR Mhealth Uhealth. 2020;8(2):e16741. PMID:32012102

29. Russell E, Lloyd-Houldey A, Memon A, Yarker J. Factors Influencing Uptake and Use of a New Health Information App for Young People. Journal of Technology in Human Services. 2018;36(4):222-40.

30. Michie S, Atkins L, West R. The Behaviour Change Wheel: A Guide to Designing Intreventions. London: Silverback Publishing; 2014.

31. Atkins L, Francis J, Islam R, O’Connor D, Patey A, Ivers N, et al. A guide to using the Theoretical Domains Framework of behaviour change to investigate implementation problems. 2017;12(1):77. PMID:28637486

32. Tong A, Sainsbury P, Craig J. Consolidated criteria for reporting qualitative research (COREQ): a 32item checklist for interviews and focus groups. International journal for quality in health care : journal of the International Society for Quality in Health Care. 2007;19(6):349-57. PMID:17872937

33. Ericsson KA, Simon HA. How to Study Thinking in Everyday Life: Contrasting Think-Aloud Protocols With Descriptions and Explanations of Thinking. Mind, Culture, and Activity. 1998;5(3):178-86.

34. Szinay D, Perski, O., Jones, A., Chadborn, T., Naughton, F. A qualitative study exploring people’s perception of factors influencing the uptake and use of health and wellbeing smartphone apps. (2020, September 18). Retrieved from osf.io/jrkd3.

35. Pope C, Mays N. Qualitative Methods in Health Research. Qualitative Research in Health Care2006. p. 1-11.

36. Benoot C, Hannes K, Bilsen J. The use of purposeful sampling in a qualitative evidence synthesis: A worked example on sexual adjustment to a cancer trajectory. BMC medical research methodology. 2016;16(1):21. PMID:26891718

37. Ritchie J, Lewis J, Nicholls CM, Ormston R. Qualitative research practice: A guide for social science students and researchers: sage; 2013.

38. Birt L, Scott S, Cavers D, Campbell C, Walter F. Member Checking: A Tool to Enhance Trustworthiness or Merely a Nod to Validation? Qualitative Health Research. 2016;26(13):1802-11.

39. Perski O, Blandford A, Ubhi HK, West R, Michie S. Smokers' and drinkers' choice of smartphone applications and expectations of engagement: a think aloud and interview study. BMC Medical Informatics and Decision Making. 2017;17(1):25. PMID:28241759

40. Hickey E, McMillan B, Mitchell C. Practitioners should embrace, not ignore, health apps. BMJ : British Medical Journal. 2015;350:h2336. PMID:25953643

41. Dennison L, Morrison L, Conway G, Yardley L. Opportunities and challenges for smartphone applications in supporting health behavior change: qualitative study. J Med Internet Res. 2013;15(4):e86. PMID:23598614

42. Lerner JS, Li Y, Valdesolo P, Kassam KS. Emotion and Decision Making. Annual Review of Psychology. 2015;66(1):799-823. PMID:25251484 
43. Perski O, Naughton F, Garnett C, Blandford A, Beard E, West R, et al. Do Daily Fluctuations in Psychological and App-Related Variables Predict Engagement With an Alcohol Reduction App? A Series of N-Of-1 Studies. JMIR Mhealth Uhealth. 2019;7(10):e14098. PMID:31579022

44. Yardley L, Morrison L, Bradbury K, Muller I. The person-based approach to intervention development: application to digital health-related behavior change interventions. J Med Internet Res. 2015;17(1):e30. PMID:25639757

45. Blank G. and Dutton WH, with Lefkowitz, J.,. Percieved Threats to Privacy Online: The Internet in Britain. Oxford Intrenet Survey 2019. Oxford Internet Institute, University of Oxford [Internet]. 2019 01.02.2020. Available from: https://oxis.oii.ox.ac.uk/wp-content/uploads/sites/43/2019/09/OxIS-report-2019final-digital-PDFA.pdf. 


\section{Supplementary Files}




\section{Figures}


A visual representation of mapping the Theoretical Domains framework onto the COM-B model.

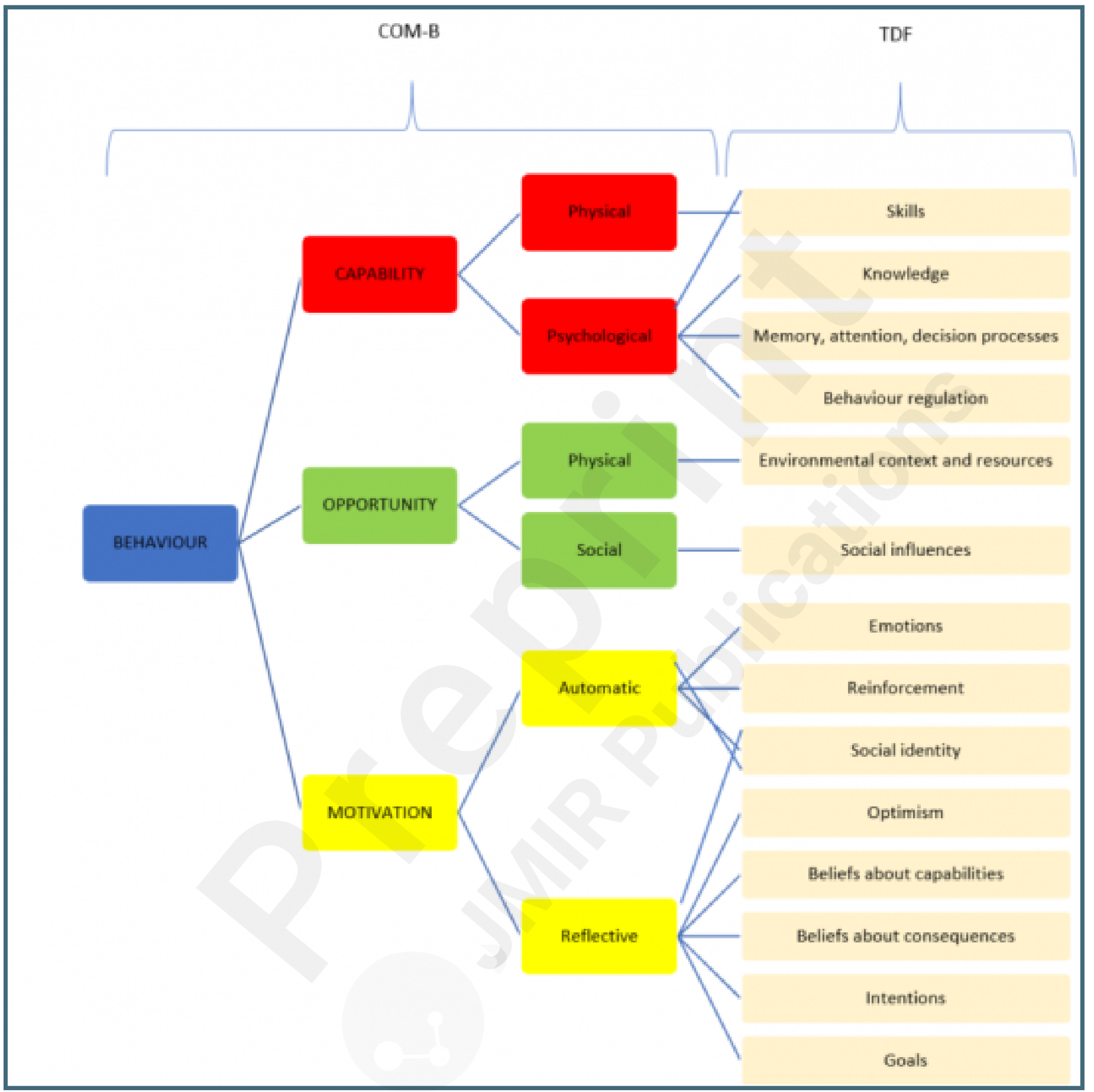


Screenshot of the Public Health England's 'One You Apps' portal and the 'NHS Apps Library'.

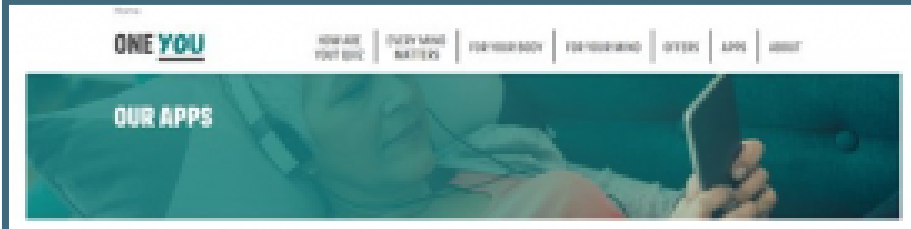

ONE vTU AWS
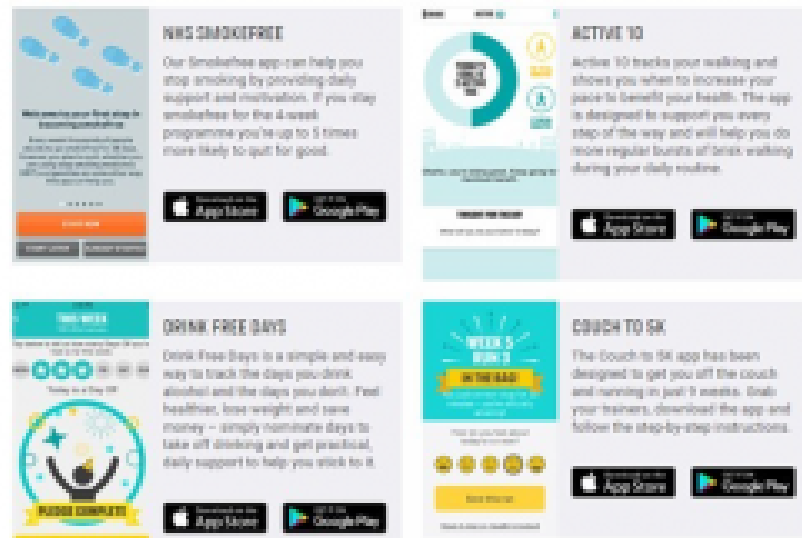

요 $=$

WHS

$-$

\section{NHS Apps Library}

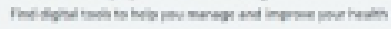

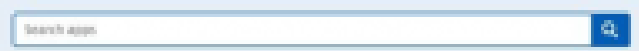

O thens

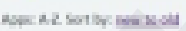

Act Dentalurinabos

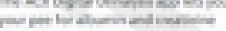

then

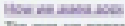

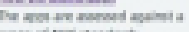

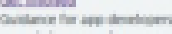

Patrendoudnotad

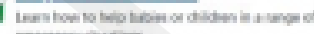
movertisen nim

Pentribatik

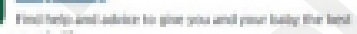
nerikis fim

(17) Mnisted

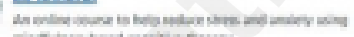

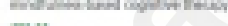




\section{Multimedia Appendixes}


Consolidate Criteria fro reporting Qualitative Studies (COREQ): 32 items checklist. URL: http://asset.jmir.pub/assets/cc6bfd305b1c8a93b2a0872a5a7af939.pdf

Screening questionnaire.

URL: http://asset.jmir.pub/assets/381e709359ba704f6c4330970619c74d.pdf

Topic Guide.

URL: http://asset.jmir.pub/assets/58d5777092323f220b559c4c6845c5f7.pdf

Participants' characteristics.

URL: http://asset.jmir.pub/assets/0198ab1a3e110bc111e97f4771d8c1a6.pdf 